\section{Epitaktische Goldaufdampfschichten im Hochvakuum}

\author{
Helmut Viefhaus*
}

Institut für Theoretische Physik der Universität Karlsruhe (Z. Naturforschg. 22 a, 2123 [1967]; eingegangen am 4. November 1967)

MÜlleR und Viefhaus hatten kürzlich ${ }^{1}$ auf ausgeheizten Glimmerspaltflächen im UHV aufgedampfte Goldschichten mittels Beugung langsamer Elektronen ${ }^{2}$ auf ihre Kristallstruktur in Abhängigkeit von der Unterlagentemperatur $T_{\mathrm{u}}$ untersucht. Ergänzend hierzu stellte ich jetzt auf Glimmerunterlagen, die wie früher an Luft gespalten, aber nicht besonders ausgeheizt waren, im Hochvakuum (HV) bei etwa $10^{-6}$ Torr unter sonst gleichen experimentellen Bedingungen wie früher zahlreiche Goldschichten von nahe $300 \AA$ Dicke bei $T_{\mathrm{u}}$ zwischen $20{ }^{\circ} \mathrm{C}$ und $450{ }^{\circ} \mathrm{C}$ her, die nach Übertragung ins Elektronenmikroskop mittels Beugung schneller Elektronen studiert wurden. Zwischen $T_{\mathrm{u}}=20^{\circ} \mathrm{C}$ und $100{ }^{\circ} \mathrm{C}$ ergaben sich polykristalline Schichten (Abb. $1^{\dagger}$ ). Darüber bis nahe $350^{\circ}$ stellte sich zunehmende Einfachorientierung mit [111] senkrecht zur Unterlage ein (Abb. 2). Ein einzelner Ring tritt stark hervor und zeigt zudem mit seinen Aufhellungen schon größere, einheitlich orientierte Kristalle an **. In der Umgebung von $T_{\mathrm{u}}=390{ }^{\circ} \mathrm{C}$ wird die Einfachorientierung vollständig (Abb. 3), nur ein Ring ist noch zu sehen. Seine 12 Aufhellungen erweisen wie in ${ }^{1}$ das Vorkommen von Kristallzwillingen. Schichten, die oberhalb etwa $420{ }^{\circ} \mathrm{C}$ aufgedampft wurden, ergaben stets Einkristallorientierung mit (111) parallel zur Unterlage (Abb.4). Bei allen diesen im HV hergestellten Goldschichten lieferten dagegen Versuche mit langsamen Elektronen wegen der starken Gasbelegung, die sich auch noch im HV in kurzer Zeit auf der Schichtoberfläche einstellt, keinerlei Beugungsbilder.

Von den neuen Ergebnissen aus dem HV ist eine Auswahl mit den früheren vom UHV $^{1}$ zusammen in Abb. 5 durch die zwei durchgehenden, waagerechten Punktreihen dargestellt ***.

Die kristallographische Struktur wurde jeweils durch unterschiedliche Punktzeichen kenntlich gemacht. Sowohl im HV wie im UHV lassen sich also mit genügend wachsender Unterlagentemperatur nach verschiedenen Zwischenstufen von Mischorientierung Einkristallschichten erzeugen. Aber die Bereiche entsprechender Struktur liegen für die UHV-Versuche bei erheblich größeren Temperaturen als für die HV-Versuche.

Neben den eigenen Messungen sind in die Abb. 5 zum Vergleich auch Ergebnise aus der Literatur ${ }^{3-6}$

* Jetzt Institut für Technische Chemie der Technischen Universität Berlin.

1 K. Müller u. H. Viefhaus, Z. Naturforschg. 21 a, 1726 [1966].

2 K. Müller, Z. Phys. 195, 105 [1966].

* Die isolierten, scharfen Punktreflexe stammen von der Glimmerunterlage, die hier nicht völlig entfernt war.

*** Die Druckangaben $P_{\mathrm{D}}$ beziehen sich auf den Vorgang des Aufdampfens der Schichten. Danach liegt der Druck im UHV bei $10^{-9}$ Torr.

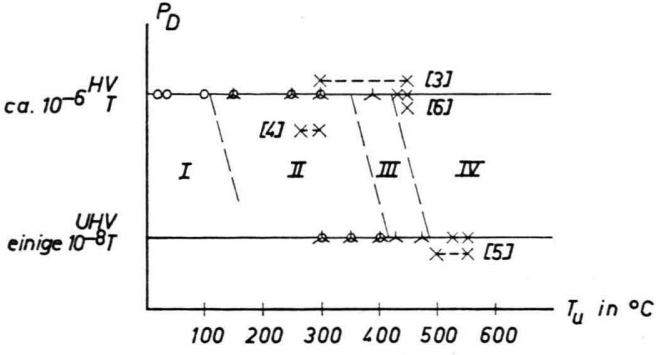

$\begin{aligned} \text { Bereich } & \text { o polykristallin } \\ & \text { II polykristallin + Einfachorientierung } \\ \text { III Einfachorientierung } & \text { II } \times \text { einkristallisch }\end{aligned}$

Abb. 5. Strukturen in Abhängigkeit von Druck und Temperatur.

über epitaktische Goldeinkristallschichten auf Glimmer eingetragen. Leider sind hiervon die Versuchsbedingungen nicht genau bekannt.

Im UHV stimmen unsere Ergebnisse mit denen von Hines $^{5}$ überein. Die Goldatome müssen hier offenbar wegen sehr tiefer Potentialmulden des Glimmers durch Temperaturerhöhung hinreichend Energie erhalten, um die für das epitaktische Wachstum notwendige Oberflächenbeweglichkeit zu erreichen.

Die neuen Ergebnisse der vorliegenden Mitteilung bestätigen zunächst die in der vorausgegangenen ${ }^{1}$ durch Vergleich mit der Literatur gewonnene Annahme, daß saubere Oberflächen (im UHV) bedeutend höhere Epitaxietemperaturen benötigen als belegte (im HV). Weiterhin deutet ein Vergleich mit den Ergebnissen von Adamsky und LeBlanc ${ }^{3}$ darauf hin, daß die niedrigsten Unterlagentemperaturen bei solchen Oberflächen zu erwarten sind, die durch Spalten im HV erzeugt wurden (s. Abb. 5). Zu dieser Ansicht gelangt auch Pashley in seinem zusammenfassenden Artikel über Epitaxie ${ }^{7}$. Auch die alte Arbeit von RüDIger ${ }^{6}$, der an Luft spaltete, paßt in unser Bild. Dagegen lassen sich die Ergebnisse von Chopra u. a. ${ }^{4}$ nicht ohne weiteres einordnen. Obwohl hier an Luft gespalten wurde, liegen die Punkte in Abb. 5 bei kleineren Temperaturen als die Vakuumergebnisse von ${ }^{3}$. Ob hierfür allein das bessere Vakuum von $10^{-7}$ Torr bei Chopra u. a. verantwortlich ist, ob der gespaltene Glimmer nur unter kurzer Zwischenbelüftung in die Vakuumapparatur eingebracht wurde, oder welche Gründe sonst vorliegen, muß offengelassen werden.

Herrn Prof. Dr. Franz Wolf, der die Arbeit ermöglichte, und Herrn Dr. KLAus Müller danke ich für ständige Förderung aller Art, Herrn Dr. Ludwig Albert für elektronenmikroskopische Aufnahmen und der Deutschen Forschungsgemeinschaft für die Bereitstellung von Mitteln.

3 R. F. Adamsky u. R. E. LeBlanc, J. Vac. Sci. Techn., Vol. 2, 79 [1965].

4 K. L. Chopra, L. C. Bobb u. M. H. Francombe, J. Appl. Phys. 34 (No. 6), 1699 [1963].

5 R. L. Hines, J. Physique 25, 134 [1964].

6 O. Rüdiger, Ann. Phys. 30, 505 [1937].

7 D. W. Pashley, Adv. Phys. 14, 327 [1965].

$\dagger$ Abb. 1-4 auf Tafel S. 2122 b. 